This Section of Epidemiology and Psychiatric Sciences regularly appears in each issue of the Journal to cover methodological aspects related to the design, conduct, reporting and interpretation of clinical and epidemiological studies. The aim of these Editorials is to help developing a more critical attitude towards research findings published in international literature, promoting original research projects with higher methodological standards, and implementing the most relevant results of research in every-day clinical practice.

Corrado Barbui, Section Editor and Michele Tansella, Editor EPS

\title{
What are neuroimaging meta-analytic procedures?
}

\author{
S. Cortese ${ }^{1,2 *}$, F.X. Castellanos ${ }^{1,3}$ and S.B. Eickhoff ${ }^{4,5}$ \\ ${ }^{1}$ Phyllis Green and Randolph Cowen Institute for Pediatric Neuroscience, Child Study Center of the NYU Langone Medical Center, New York, \\ NY, USA \\ ${ }^{2}$ Child Neuropsychiatry Unit, Department of Life Sciences and Reproduction, Verona University, Verona, Italy \\ ${ }^{3}$ Nathan S. Kline Institute for Psychiatric Research, Orangeburg, NY, USA \\ ${ }^{4}$ Institute of Neurosciences and Medicine (INM-1), Research Centre Juelich, Juelich, Germany \\ ${ }^{5}$ Institute of Clinical Neuroscience and Medical Psychology, Heinrich Heine University, Düsseldorf, Germany
}

Activation likelihood estimation (ALE) meta-analyses allow investigators to integrate the results of multiple neuroimaging studies, potentially yielding novel results that may not have been evident in the individual studies. Here, we provide a brief, introductory description of ALE methods for readers without extensive expertise in neuroimaging.

Received 18 December 2012; Revised 9 January 2013; Accepted 9 January 2013; First published online 12 February 2013

Key words: activation likelihood estimation, meta-analysis, neuroimaging.

The application of neuroimaging techniques to the investigation of psychiatric disorders has generated a burgeoning literature in the last two decades. Owing to the heterogeneity of sample characteristics (e.g., sample size, sex ratio, age range, inclusion of comorbidities) and analytical procedures (e.g., experimental paradigms, statistical approaches) (Purgato \& Adams, 2012), different neuroimaging studies assessing a particular brain process or mental disorder often yield heterogeneous results. As a way to possibly address this challenge, neuroimaging meta-analytic tools have been introduced, allowing researchers to integrate findings from individual neuroimaging studies and identify those brain regions showing the investigated effect robustly over multiple experimental settings and samples. Among neuroimaging metaanalytic procedures, activation likelihood estimation

* Address for correspondence: Dr S. Cortese, NYU Child Study Center, One Park Avenue, New York, NY 100161, USA.

(Email: samuele.cortese@gmail.com)
(ALE) (Turkeltaub et al. 2002; Eickhoff et al. 2009, 2012) represents the most commonly used approach.

We will primarily consider the meta-analysis of functional magnetic resonance imaging (fMRI) studies, although the same concepts and methods apply to other types of MRI data (e.g., structural MRI analysed by a particular approach called 'voxel-based morphometry') or to investigations using other neuroimaging techniques (e.g., positron emission tomography). The purpose of an fMRI study is to identify the brain regions that undergo changes in activity (either increase or decrease) during a task that is performed while the subject lies in the MRI scanner (e.g., pressing a button when the colour of a visual stimulus changes, to assess attention functions). When applied to the investigation of psychiatric disorders, the goal of fMRI studies is usually to identify those brain regions which are hypo- or hyper-active in patients relative to healthy individuals (or, more rarely, relative to patients with other disorders) (Chiesa et al. 2011; Bellani et al. 2012).

Importantly, neuroimaging results almost always report tables listing the location in the brain of the 
(a)

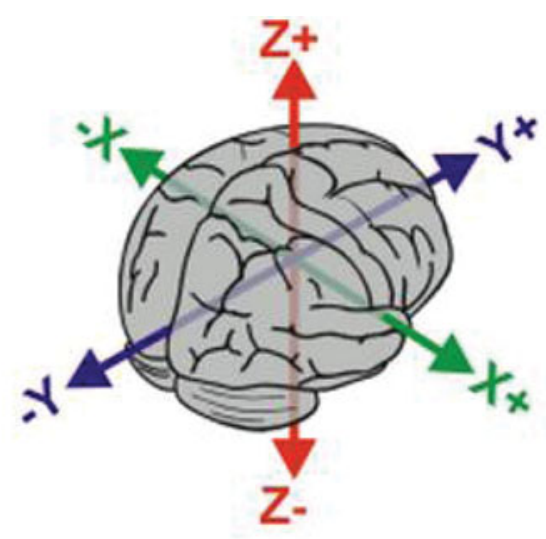

(b)

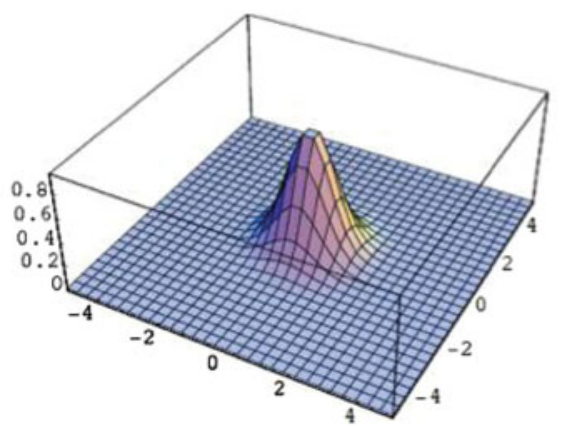

(c)

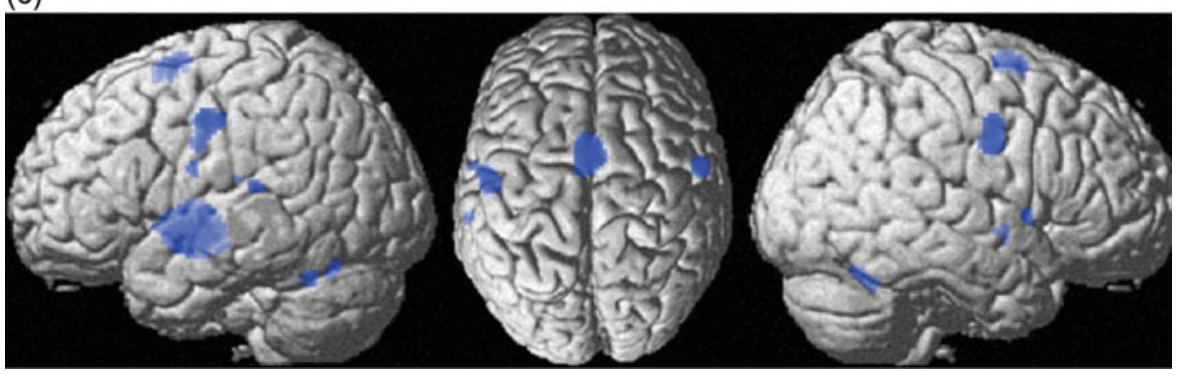

Fig. 1. (a) Tridimensional brain coordinates system. (b) Tridimensional Gaussian distribution. (c) Hypothetical example of an ALE meta-analysis of fMRI studies. Coloured areas might represent, for example, brain areas that are significantly more activated in individuals with a particular psychiatric disorder relative to comparisons across all studies retained in the meta-analysis. (A colour version of this figure is available online at http://journals.cambridge.org/eps)

peaks of hypo- or hyper-activation (the so-called 'foci' of differential activation) as three-dimensional coordinates, i.e., with reference to spatial axes in a standard reference space (Fig. 1a). ALE builds on this high degree of standardization to statistically evaluate the brain locations in which the convergence of reported activations across studies is higher than would be expected by chance. In the clinical context, the fundamental question in ALE is thus: 'Where have hyper- or hypo-activation foci in a particular disorder consistently been reported across studies?' In evaluating this question, it is important to take into account that the coordinates reported in MRI studies are associated with some degree of spatial uncertainty (for a more detailed discussion on the factors contributing to this uncertainty, cf. Eickhoff et al. 2009). The key idea behind ALE is thus to represent the foci reported in the individual studies not as 'exact' points but rather by treating them as centres of a tridimensional Gaussian probability distribution (Fig. 1b), with the centre indicating the highest probability of activation. This procedure is performed for each focus of each study included in the meta-analysis and yields a probabilistic location of the effects reported in that particular study. Afterwards, the probabilistic data are pooled across all individual studies. This is done separately for each volume unit in the brain, known as a 'voxel', by computing for each voxel the union of these probabilistic values, denoting how likely it was the true location of a focus across all studies. The value obtained with this procedure is termed the 'activation likelihood estimation (ALE' for that particular 
voxel, and quantifies the probabilistic convergence across experiments.

Finally, the issue is to differentiate random convergence, i.e., overlap that occurs by chance, from that which reflects consistent findings across studies. To this purpose, ALE values in each voxel are compared with the values that would be obtained under the nullhypotheses of random spatial association. That is, we simulate what ALE values would look like if any spatial convergence was just random and then only retain those values that are highly unlikely to arise by chance, i.e., which exceed a certain statistical threshold. From this approach, it follows that ALE can be applied only to studies that explore the whole brain, since studies focusing on a priori regions of interest (ROIs) violate the assumption that the likelihood of activation, under the null hypothesis, is equal in every part of the brain. Finally, the output of the ALE procedure, indicating regions of above-chance convergence across studies, is visualized on a brain template where areas of consistent hyper- or hypo-activation across studies are indicated with colours (Fig. 1c).

The application of ALE has allowed pooling data to better understand the neural correlates of several brain processes [e.g., working memory (Rottschy et al. 2012)] or psychiatric disorders [e.g., schizophrenia (Minzenberg et al. 2009); attention-deficit hyperactivity disorder (Cortese et al. 2012)]. It thus allows investigators to draw more generalizable conclusions about the locations of physiological and pathological processes. More detailed information on ALE can be found at: http://www.brainmap.org/ale/.

\section{Declaration of Interest}

No financial support was received for this paper. The authors declare no financial involvement/affiliation with any organization whose financial interests may be affected by material in the manuscript.

\section{References}

Bellani M, Garzitto M, Brambilla P (2012). Functional MRI studies in disruptive behaviour disorders. Epidemiology and Psychiatric Sciences 21, 31-33.

Chiesa A, Brambilla P, Serretti A (2011). Neuroimaging of mindfulness meditations: implications for clinical practice. Epidemiology and Psychiatric Sciences 20, 205-210.

Cortese S, Kelly C, Chabernaud C, Proal E, Di Martino A, Milham MP, Castellanos FX (2012). Toward systems neuroscience of ADHD: a meta-analysis of $55 \mathrm{fMRI}$ studies. American Journal of Psychiatry 169, 1038-1055.

Eickhoff SB, Laird AR, Grefkes C, Wang LE, Zilles K, Fox PT (2009). Coordinate-based activation likelihood estimation meta-analysis of neuroimaging data: a random-effects approach based on empirical estimates of spatial uncertainty. Human Brain Mapping 30, 2907-2926.

Eickhoff SB, Bzdok D, Laird AR, Kurth F, Fox PT (2012). Activation likelihood estimation meta-analysis revisited. Neuroimage 59, 2349-2361.

Minzenberg MJ, Laird AR, Thelen S, Carter CS, Glahn DC (2009). Meta-analysis of 41 functional neuroimaging studies of executive function in schizophrenia. Archives of General Psychiatry 66, 811-822.

Purgato M, Adams CE (2012). Heterogeneity: the issue of apples, oranges and fruit pie. Epidemiology and Psychiatric Sciences 21, 27-29.

Rottschy C, Langner R, Dogan I, Reetz K, Laird AR, Schulz JB, Fox PT, Eickhoff SB (2012). Modelling neural correlates of working memory: a coordinate-based meta-analysis. Neuroimage 60, 830-846.

Turkeltaub PE, Eden GF, Jones KM, Zeffiro TA (2002). Meta-analysis of the functional neuroanatomy of single-word reading: method and validation. Neuroimage 16, 765-780. 